\title{
Evaluation of Merge Control Strategies at Interstate Work Zones under Peak and Off-Peak Traffic Conditions
}

\author{
Ossama E. Ramadan, Virginia P. Sisiopiku \\ Department of Civil, Construction, and Environmental Engineering, University of Alabama at Birmingham, \\ Birmingham, USA \\ Email: oramadan@uab.edu
}

Received 23 March 2016; accepted 15 April 2016; published 18 April 2016

Copyright (C) 2016 by authors and Scientific Research Publishing Inc.

This work is licensed under the Creative Commons Attribution International License (CC BY).

http://creativecommons.org/licenses/by/4.0/

(c) (i) Open Access

\begin{abstract}
Maintenance and rehabilitation projects of interstate facilities typically mandate lane closures. Lane closures require merging maneuvers that often result in reduced speeds and traffic bottlenecks. Work zone impacts on traffic operations are magnified when project durations are extended. Conventionally, work zone traffic control plans are developed to address work zone impacts. This study evaluated various merge control strategies at interstate work zones peak and off-peak traffic conditions and summarized related impacts. A comprehensive microscopic simulation model was developed in full consideration of driver/vehicle behavior at work zones. The analysis of simulation results revealed that merge control strategies, when implemented during peak and off-peak conditions, can preserve the level of service and provide favorable mobility, safety, and environmental impacts. In addition, results indicate that transportation agencies' practice of scheduling work zone activities during the off-peak may not be the most optimum approach. Overall, the findings of this study highlight the need for evaluation of work zone scheduling practices in full consideration of agency, user, and project costs.
\end{abstract}

\section{Keywords}

Traffic Simulation, Bottlenecks, Work Zone Traffic Control, Traffic Congestion

\section{Introduction}

Highway maintenance and rehabilitation projects are essential for maintaining the nation's infrastructure functionality. Such projects often mandate lane closures to provide a work space, which in turn result in bottlenecks 
and disruption of traffic [1]. Studies indicate that work zones represent the second largest cause of nonrecurring delay on principal arterials with nearly $24 \%$ of all nonrecurring delay attributed to work zone operations [2].

In an effort to reduce undesirable impacts from work zone presence, transportation agencies opt to schedule maintenance activities during off-peak hours. However, such practice may lengthen project duration and increase maintenance-related costs as a result of repetitive setup, frequent cessation, and associated costs to nighttime operations. This is worrisome, especially given the fact that transportation agencies are often pressured to reduce the time they spend on construction to minimize the impact on the traveling public and local businesses [3].

Another common practice is the use of traffic control to facilitate vehicle movement upstream and through the work zone. Conventional traffic control plans normally work well when traffic demand is less than the capacity of the open lanes. However, when demand exceeds capacity, both traffic operations and safety at work zone locations are compromised, especially when the congestion extends upstream beyond the advance lane closure signs [4].

A need exists to evaluate various merge control strategies at interstate work zones under peak and off-peak traffic conditions and determine optimal practices for scheduling of construction activities that mitigate bottleneck effects at work zones while also considering project costs and scheduling efficiency constraints.

\subsection{Background}

Transportation agencies rely on traffic control plans to minimize the traffic impacts from work zone presence. Most agencies currently follow the conventional temporary traffic control strategies set by Part 6 of the Manual on Uniform Traffic Control Devices (MUTCD) [5]. In an earlier study, the authors [6] performed a comprehensive literature review and a national survey of practices where they identified alternative traffic control strategies that hold promise toward improving effectiveness as compared to conventional merge control. Such alternative control strategies were categorized in that study as follows:

- Late merge control, which encourages drivers to retain their lanes until they reach the lane closure taper. Late merge is sometimes referred to as "zipper merge" when associated with an alternating "zipper" fashion merge maneuver into the open lane(s). Late merge control has been identified as the second most implemented bottleneck merge control strategy at interstate work zones.

- Early merge control, which encourages drivers to merge into open lane(s) sooner than they would do, by providing advance signs ahead of lane closure(s) or merge tapper(s). Early merge control has been identified as the most implemented bottleneck merge control strategy for interstate work zones.

- Mainline merge metering strategies, which are built upon the same architecture as late merge control; however, a merge meter, similar to a conventional ramp meter, is installed at the tapper or point of merge to regulate the release of vehicles trying to merge into open lanes. Literature review indicated that mainline merge metering is not implemented by transportation agencies in the US.

- Temporary ramp metering, which builds upon the well-established benefits of ramp metering as an effective strategy for improving traffic flow along the mainline by controlling the rate of vehicles entering from the on-ramp into the mainline traffic. The documented mobility and productivity benefits of ramp meters motivated researchers to study the use of temporary ramp meters as an alternative to managing traffic on interstate work zones located near or at on-ramps. Literature review indicated that temporary ramp metering is not currently implemented by any transportation agency in the US.

\subsection{Problem Statement and Research Objective}

Earlier literature review and a national survey of agencies practices indicated that there are four alternative bottleneck merge control strategies for interstate work zones [6]. However, two of the identified strategies are still a matter of research, indicating a gap between research and practice. In their earlier literature review and survey of practices, the authors noticed that all existing studies evaluate these bottleneck merge control strategies only under off-peak conditions. Moreover, literature and agency practices indicate the lack of formal guidance on selection and assessment of relevant impacts for the identified strategies. In addition, available literature lacks a comprehensive comparison between these four strategies. Accordingly, there is a need to study and evaluate the identified bottleneck merge control strategies at work zones, to better understand their impacts and benefits, and to provide some guidance to agencies for selecting an appropriate strategy. 
The objectives of this study are (a) to compare the previously identified four strategies at interstates work zones of various lengths under peak and non-peak traffic conditions, and (b) report their associated impacts on traffic operations and the environment. The stated objectives can be achieved by providing answers to the following questions: What are the impacts of each strategy? Which strategy provides the most benefits? And, is scheduling of work zone activities during off-peak periods justified?

\section{Methods}

The focus of this study is to consistently capture detailed elements of traffic control within the immediate work zone area. According to the comprehensive work zone modeling and simulation study that was performed by Hardy and Wunderlich [7], the most appropriate methodology would be a mono-scale method that utilizes microscopic simulation. Therefore, this study used of Corridor Simulation (CORSIM) version 6.3 for developing a stochastic microscopic simulation model of a section of I-65 in Birmingham, Alabama, as shown in Figure 1. This is the latest version of CORSIM that was released by McTrans Center at the University of Florida for the US Federal Highway Administration (FHWA). The study segment is approximately 14 miles (22.53 Km) in length and extends from exit 247, where it intersects Valleydale Road, to exit 261, where it intersects Interstate 20/59 (I-20/59). The study segment typically has three 12-ft (3.66 m) lanes per mainline direction, with auxiliary lanes added at ramps locations. The posted speed limit on this study interstate is generally $60 \mathrm{mph}(96.6 \mathrm{~km} / \mathrm{h})$ with an advisory speed limit of 45 mph (72.45 km/h) on ramps. The 2014 Annual Average Daily Traffic (AADT) for this study section is 122,510 veh/day, with peak traffic typically occurring on weekdays between 7:00 am and 9:00 am, and off-peak traffic typically occurring between 2:00 am and 4:00 am [8]. The following subsections illustrate base model development, base model calibration, setting up the microsimulation platform for work zone simulation, and simulation of each of the identified four merge control strategies.

\subsection{Base Model Development}

Two base models were developed to simulate typical existing, or baseline, peak and off-peak traffic conditions. To develop these base models, data related to road geometry, pavement conditions, traffic volumes, and traffic characteristics were collected. These data were collected during the period February 2014 to February 2015 at the beginning of the study segment, at each entry and exit ramp, at mid-sections between ramps or where geometry would change, and at the end of the study segment. This study followed the guidelines and methods stated by the FHWA Traffic Analysis Tool Box, Volumes III, IX, and IX [7] [9] [10] and the Institute of Transportation Engineers (ITE) Manual of Transportation Engineering Studies [11] to setup and calibrate CORSIM. To check the quality of the base models, a review of physical inputs and traffic volumes was conducted to identify any errors that may have occurred while developing the base models. The review did not identify any errors or non-conformities in the physical or traffic inputs including acceleration/deceleration lane lengths; number of lanes and lane alignment; lane drops/lane adds; free flow speeds; curvature; grades; freeway volumes for each time period balance; and overall freeway volumes balance.

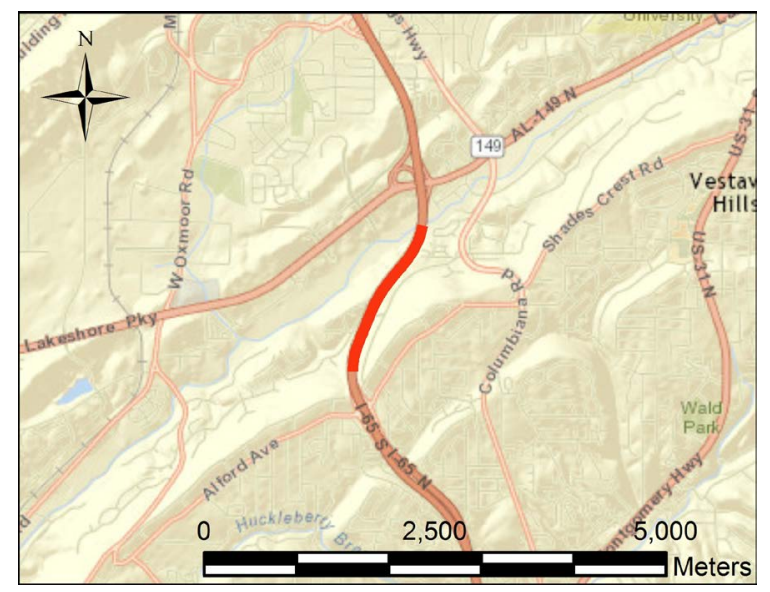

Figure 1. Map showing study area. 


\subsection{Base Model Validation and Calibration}

CORSIM has been extensively validated by several studies over the past 45 years [10]. Earlier studies provide confidence in the overall model ability to predict traffic performance such as traffic volumes, travel times, average speeds, and average delays. However, any base model has to be calibrated for local conditions so that the simulated volumes and speeds would be within of $15 \%$ of actual volumes and speeds [10]. Calibration data composing of average speeds and link volumes were collected within uniform sections along the study segment during the period February 2014 to February 2015. Speed data were collected by test vehicle method equipped with after-market GPS units. Volume data were obtained by manual observation using a handheld electronic count board. Simulated volumes and speeds were compared against field collected volumes and speeds, relative errors were calculated using Equation (1), and were plotted for each simulation period comprising 15 minutes interval. The calibrated model produced traffic volumes and speeds within a range of $\pm 10 \%$, with slightly higher traffic volumes than those of the actual facility, and slightly lower speeds than actual speeds, as illustrated by Figure 2. The base model calibration yielded satisfactory results and provided confidence in the model's ability to replicate closely actual conditions.

$$
\text { Relative Error }=\frac{\text { Simulated Data }- \text { Measured Data }}{\text { Measured Data }} \times 100
$$

Because CORSIM is a stochastic time-step microsimulation model, the Output Processor was configured to use the same random number seeds across all simulation runs. In addition, mean cumulative outputs and corresponding standard deviations were collected from 42 scenarios that were run five times each with a total of 210 simulation runs. Manipulating CORSIM for simulating driving conditions in work zones has been extensively studied by other researchers, and seven parameters were identified along recommended values to setup CORSIM for work zone driving conditions independent of location or geometry [7]-[10] [12] [13]. These seven parameters are:

1) Vehicle entry headway: This is the method CORSIM will use to generate vehicles at entry nodes. A negative exponential distribution was used in this study, which can be achieved by setting CORSIM to use an Erlang Distribution with $\mathrm{a}=1$.

2) Rubberneck factor: The rubberneck factor, in a percentage, represents the reduction in capacity that results from the tendency of drivers at work zones who to slow down to see what is happening. Literature recommend that open lanes at work zones as well as a portion equal to work zone length of the closed lane(s) post work zones should be coded for rubber necking incidents with a factor of $20 \%$ to account for such phenomenon.

3) Car following sensitivity multiplier: CORSIM uses driver sensitivity to account for the car-following logic. Despite the fact that drivers tend to be more aggressive at work zones, literature recommends using the default car-following sensitivity multipliers.

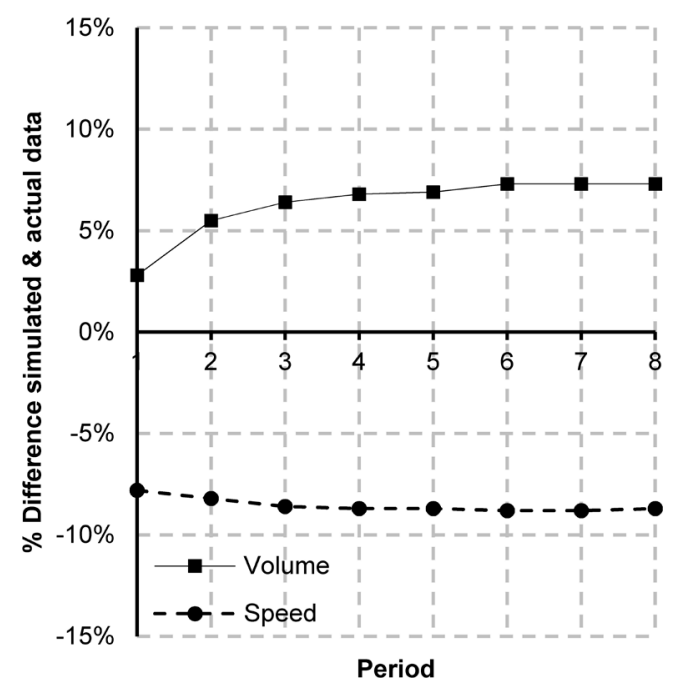

Figure 2. Speed and volume differentials for calibrated base model. 
4) Mean start-up delay time: This is a link-specific parameter that is used by the model to discharge vehicles from a ramp meter onto the freeway. The default value is 1.0 second, and literature recommends a value of 2.4 seconds when simulating work zone conditions.

5) Time to complete lane-change maneuver: This parameter specifies the time needed by a driver to complete a lane-change maneuver. The default value is 2.0 seconds, and a literature recommended using a value of 2.2 seconds when simulating work zones to account for the increased driver aggressiveness at work zones, which does not usually allow drivers to complete a lane change without getting some resistance from other drivers.

6) Percent drivers yielding to merging vehicles: This parameter specifies the percentage of drivers desiring to yield the right-of-way to lane-changing vehicles attempting to merge ahead of them. The default value is $20 \%$, and literature recommended a value of $15 \%$ to account for work zone conditions.

7) Minimum separation for generation of vehicles: This parameter governs the maximum rate at which vehicles can be emitted onto the network. The default value of 1.6 seconds is appropriate for freeway operations; however, earlier studies proved that a value of 1.3 seconds will enhance the calibration of CORSIM for work zone conditions.

The following sub-sections explain how each strategy was modeled in CORSIM in the study herein.

Microscopic simulation of late merge control. Late merge control strategy depends on urging drivers to remain in the lane(s) that are about to be blocked until they reach the beginning of the tapper. This strategy attempts to consume all available capacity to accommodate as many vehicles as possible. Simulation was performed using the CORSIM built-in Incident Management Algorithm, for the aforementioned hypothetical work zone configurations. To account for the work zone presence, two incidents were coded. The first incident is a blockage on the rightmost lane starting from the beginning of simulation and extending to 2.5 hours to account for the warm-up time, and $20 \%$ rubbernecking-only on the middle lane and the left lane. The second incident is coded on the segment following the work zone and with the same length with $20 \%$ rubbernecking-only on the rightmost lane. Figure 3 provides a screen capture in CORSIM illustrating the microsimulation setup for late merge control.

Microscopic simulation of early merge control. Early merge control strategy depends on urging drivers to merge into open lane(s) as early as possible in a manner that minimizes lane changes near or at work zones. This strategy is typically used for lower volume facilities. Simulation was performed using CORSIM as a lane drop for the rightmost lane and a lane add following the work zone. In addition, rubbernecking-only incidents were coded along the work zone for open lanes to account for the speed reduction that occurs in work zones. Similar to late merge control, the model accounted for a buffer space; however, the merge taper was not accounted for as the model considered an early merge effective $5800 \mathrm{ft}(1769.00 \mathrm{~m})$ upstream of work zone. The distance of 5800 $\mathrm{ft}(1769.00 \mathrm{~m})$ accounts for merging signs placed $800 \mathrm{ft}(244.00 \mathrm{~m})$ upstream of work zone and every $1000 \mathrm{ft}$ $(305.00 \mathrm{~m})$ thereof. Figure 4 provides a screen capture in CORSIM illustrating the microsimulation setup for early merge control.

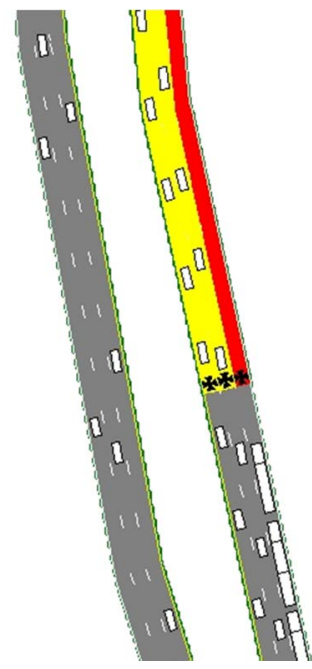

Figure 3. Work zone setup in CORSIM using late merge control. 


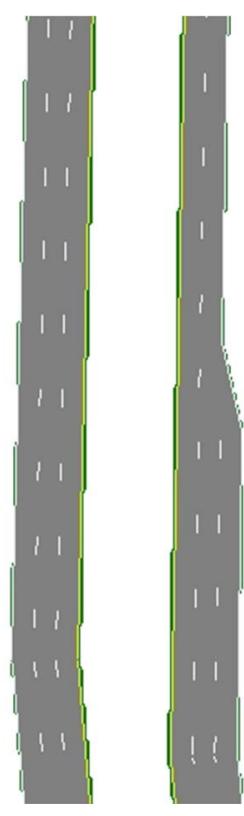

Figure 4. Work zone setup in CORSIM using early merge control.

Mainline merge metering microscopic simulation model. Mainline merge metering incorporates a merge meter that functions in the same manner as a ramp meter but is installed on the mainline of the freeway lane that is about to be dropped and vehicles have to merge in the adjacent open lane(s). The simulation model was coded to account for a mainline meter upstream of the work zone buffer zone, with an acceleration/merge lane of $300 \mathrm{ft}$ (91.50 m) [14]. The configured meter was a demand/capacity meter with capacity of 2300 veh/hr/lane [15] [16]. The CORSIM demand/capacity metering algorithm performs an evaluation of current excess capacity, immediately downstream of the metered lane, at regular intervals, based on counts from the surveillance detectors on the freeway mainline [17]. A maximum metering rate is calculated such that the capacity of this freeway section is not violated. This calculated metering rate is then applied like clock-time metering. A minimum metering rate of three green signals/60 seconds is applied to ensure that waiting vehicles are not trapped between the meter and the ramp connection to the freeway. The metering rate is also limited to headways that are greater than two seconds. Figure 5 provides a screen capture in CORSIM illustrating the microsimulation setup for mainline merge metering.

Temporary ramp metering microscopic simulation model. Temporary ramp meters are installed at on-ramps where an on-ramp feeds an interstate work zone. The purpose of the temporary ramp meter is to regulate traffic going onto the interstate hence mitigating the mobility impacts of excessive merging in such sections. A hypothetical 3-to-2 work zone was modeled on the leftmost lane downstream from the subject on-ramp. A temporary ramp meter was installed with demand/capacity algorithm similar to the one modeled in the mainline metering strategy. Figure 6 provides a screen capture in CORSIM illustrating the microsimulation setup for temporary ramp metering.

\section{Results and Discussion}

CORSIM has the capability to report several Measures of Effectiveness (MOEs) for the simulated interstate work zone configuration. These MOEs are either link-based or network-based. Link-based MOEs provide an understanding of the localized impacts of simulated merge control strategies at and in the vicinity of the work zone, while network-based MOEs capture the global impact on the simulated network. Selected MOEs were collected and averaged over five runs for each scenario while preserving the random seeds and are discussed in the following sections. Results were compared against preconstruction, or baseline conditions, conditions. Figures 7-13 illustrate the percentage change from preconstruction conditions for each MOE during peak and offpeak traffic. To simplify the comparison against preconstruction (baseline) conditions, an MOE Index ( $I_{\mathrm{MOE}}$ ) was calculated as illustrated by Equation (2), to represent MOEs compared to that of preconstruction conditions. 


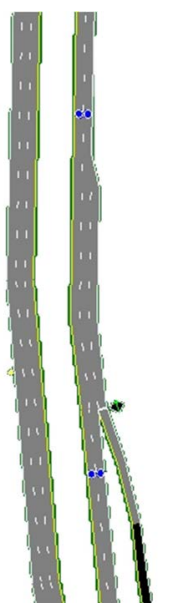

Figure 5. Work zone setup in CORSIM using mainline merge metering.

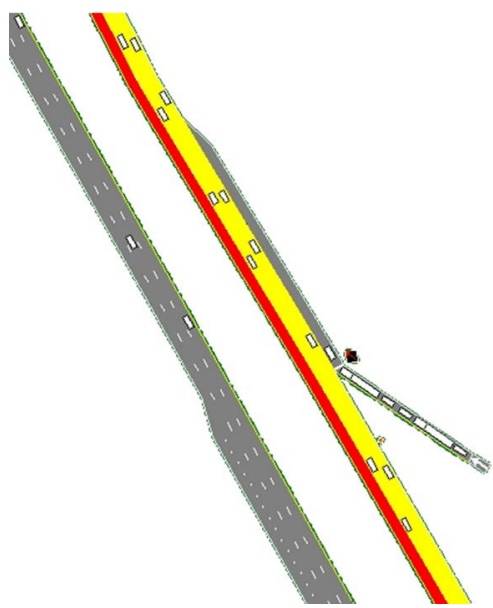

Figure 6. Work zone setup in CORSIM using temporary ramp metering.

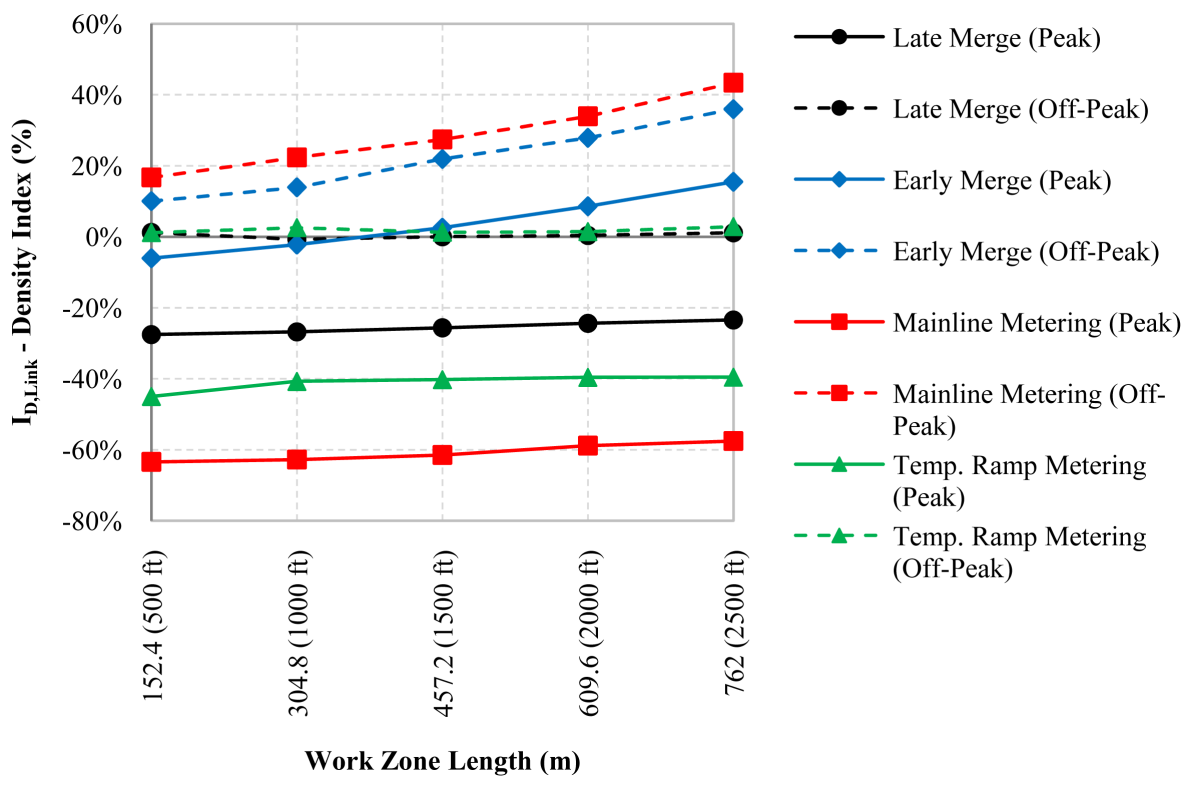

Figure 7. Density index for each merge control strategy by work zone length. 


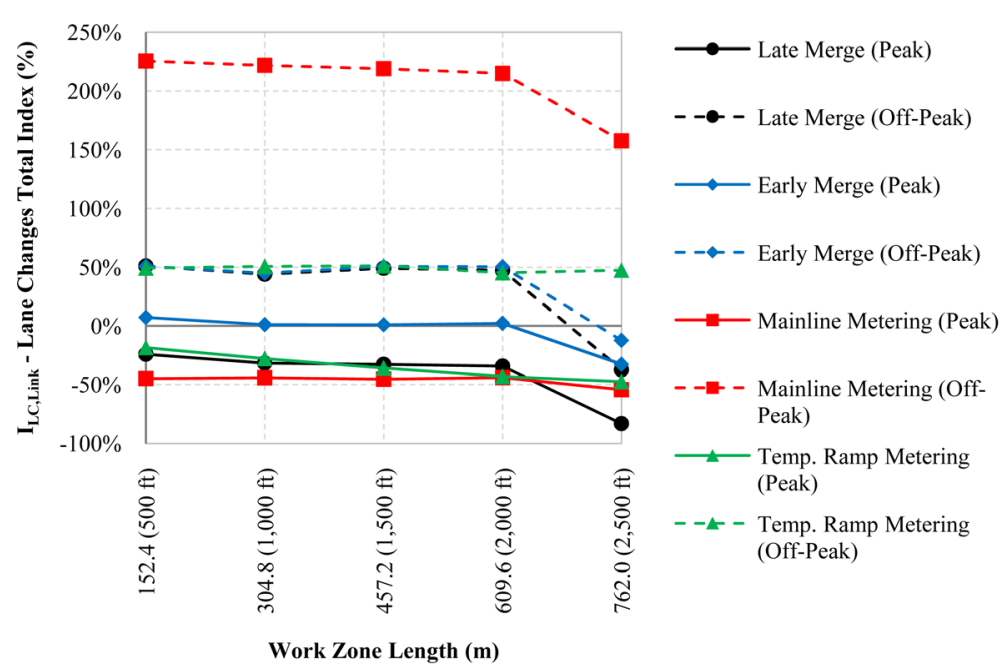

Figure 8. Lane changes index for each merge control strategy by work zone length.

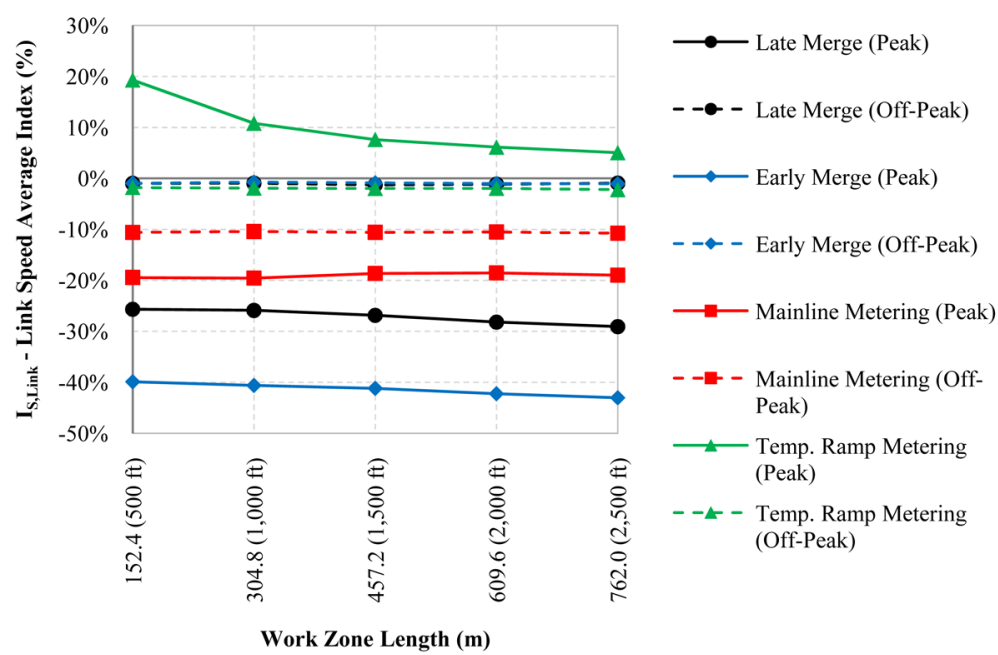

Figure 9. Link speed average index for each merge control strategy by work zone length.

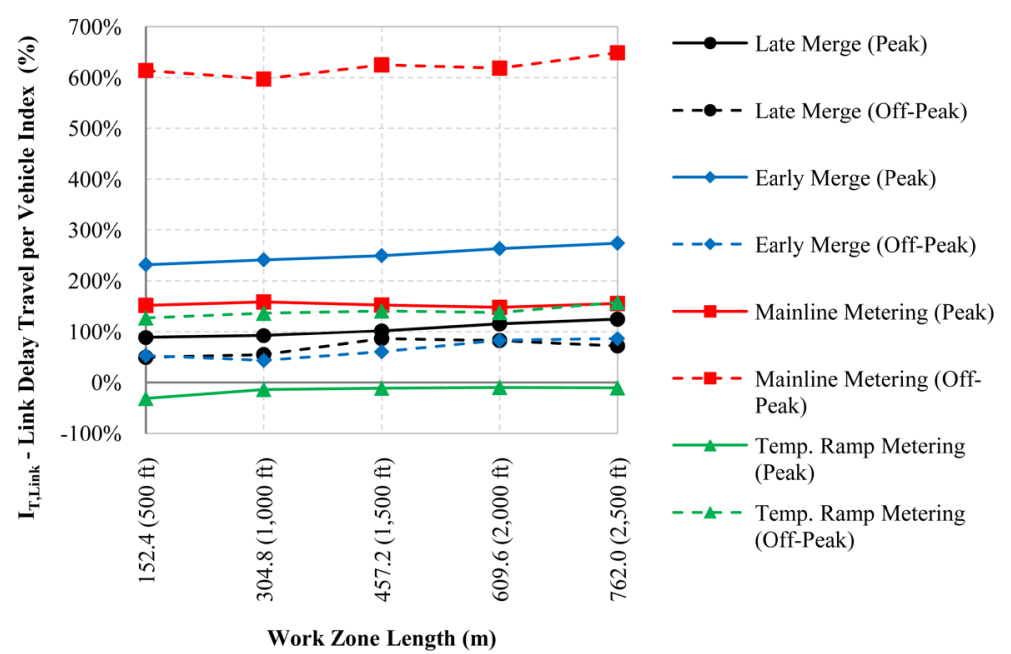

Figure 10. Link delay per vehicle index for each merge control strategy by work zone length. 


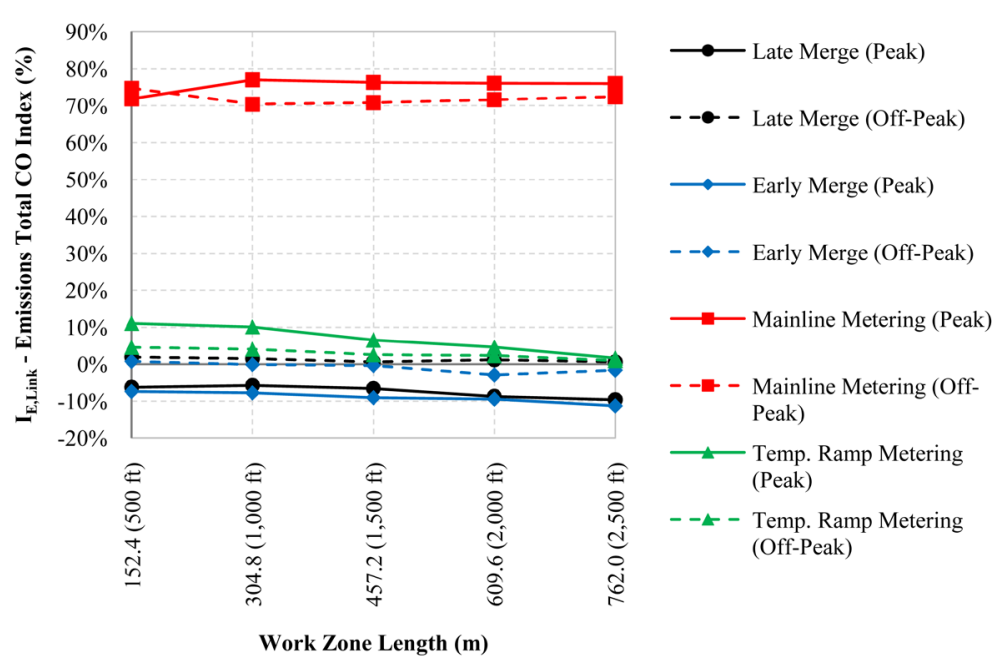

Figure 11. Link emissions total CO index for each merge control strategy by work zone length.

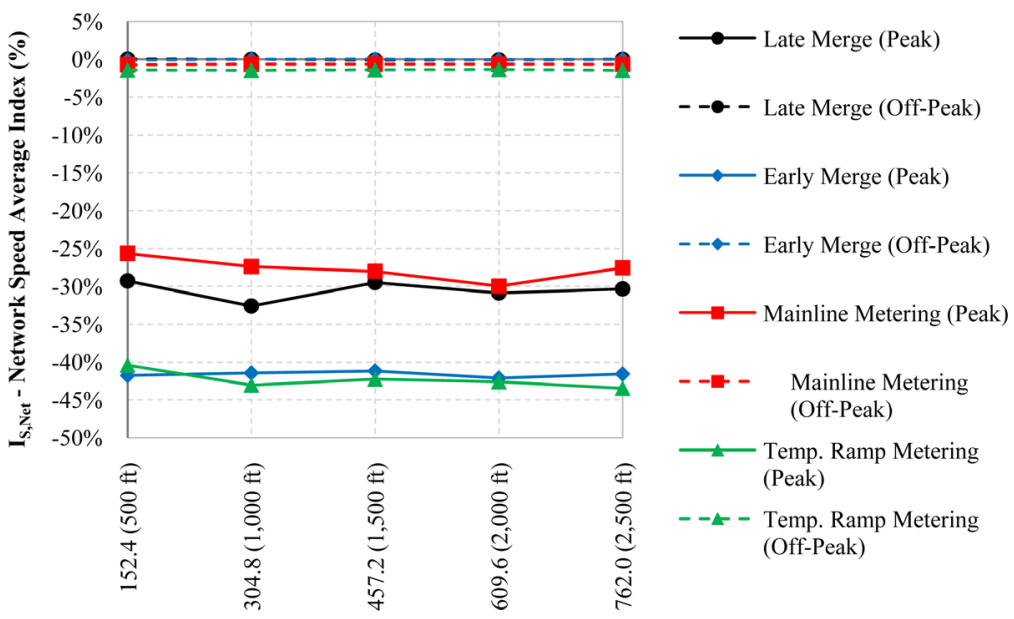

Work Zone Length (m)

Figure 12. Network speed average index for each merge control strategy by work zone length.

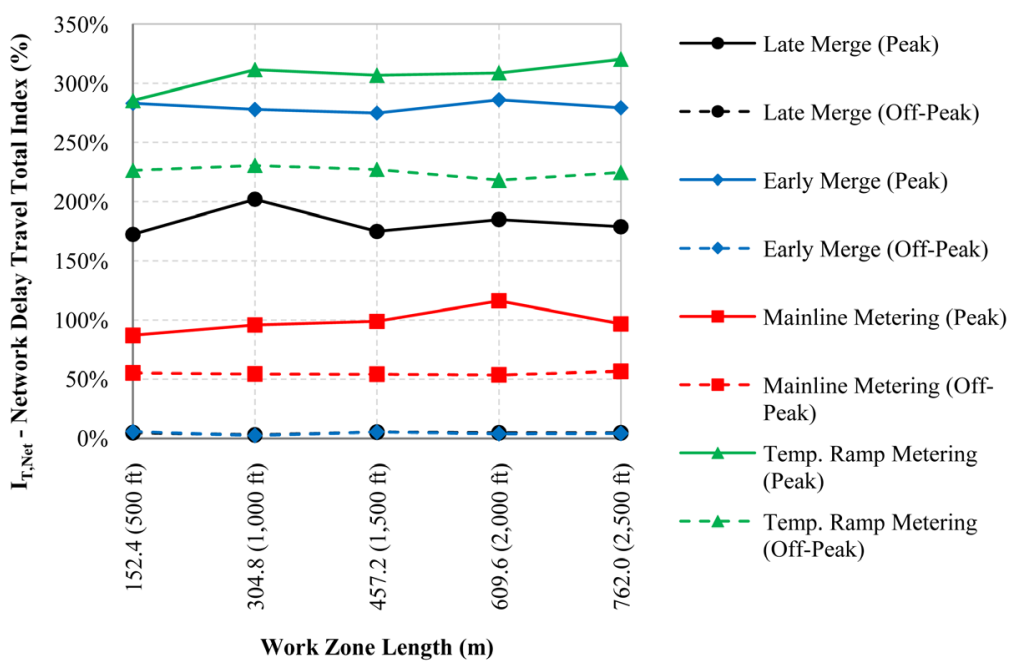

Figure 13. Network delay travel total index for each merge control strategy by work zone length. 


$$
I_{\mathrm{MOE}, i, j}=\frac{\mathrm{MOE}_{i, j}-\mathrm{MOE}_{i, P C}}{\mathrm{MOE}_{i, P C}} \times 100
$$

where, $\mathrm{MOE}_{i, P C}$ is the preconstruction MOE of the segment for a given traffic volume (i), and $\mathrm{MOE}_{i, j}$ is the same MOE for a given traffic volume $(i)$ and a given merge control strategy $(j)$. A positive $I_{\text {MOE }}$ value indicates that the facility is experiencing higher levels of that MOE compared to preconstruction conditions, and similarly a negative $I_{\mathrm{MOE}}$ value implies lower levels than that of preconstruction conditions. Preconstruction conditions were chosen as baseline conditions since the purpose of implementing any of these merge control strategies is to preserve whatever conditions existed before having a work zone.

\subsection{Link-Based MOEs}

Density per lane (vehicles/Km/lane). Density is the key measure for level of service (LOS) on freeway segments. A density index $\left(I_{\mathrm{D}, \mathrm{Link}}\right)$ was calculated, to represent density changes compared to that of preconstruction conditions. A positive $I_{\mathrm{D}, \mathrm{Link}}$ indicates that the facility is experiencing more density that preconstruction conditions, and negative $I_{\mathrm{D} \text {,Link }}$ indicates improved density. Weighted average density was used following the guidelines of the Highway Capacity Manual [18]. The density index was plotted for each merge control strategy by work zone length. Figure 7 illustrates the percentage change of density per lane from corresponding preconstruction conditions by work zone length for both peak and off-peak traffic.

It is observed that under peak traffic conditions, late merge control, temporary ramp metering and mainline merge metering yielded lower densities compared to preconstruction conditions. However, early merge control yielded improvements with shorter work zones and higher densities for longer work zones setup. This aligns with the common practice of implementing late merge control for work zone works scheduled during peak periods. Moreover, mainline merge metering yielded more promising results in terms of density. It should be noted that impacts on surface network were not evaluated when applying temporary ramp metering. Accordingly, implementation of temporary ramp metering strategies should be considered with full account for impacts over freeway and surface street networks. As for off-peak traffic conditions, late merge control and temporary ramp metering did not impact density. On the other hand mainline merge metering and early merge control yielded higher densities with mainline merge metering having the worse impact during off-peak conditions. In addition, minimal impact was found for work zone length over density, which indicates, from an operational perspective, that the merge control strategy is more important than work zone length.

Lane changes total. The total number of lane changes that have been completed during the simulation period on the links occupied by the work zone, in addition to links comprising a stretch of the interstate upstream and downstream of the work zone with the same length was considered. A lane changes total index $\left(I_{\mathrm{LC}, \text { Link }}\right)$ was calculated to represent the lane changes in comparison to those of baseline conditions. Figure 8 illustrates the $I_{\mathrm{LC}, \text { Link }}$ by work zone length for both peak and off-peak traffic. A positive $I_{\mathrm{LC}, \text { Link }}$ indicates excessive lane changing, while a negative value indicates less lane changes compared to baseline conditions.

It is observed that lane changes tend to decrease during peak traffic and as the work zone gets longer. However, during off-peak traffic, lane changes tend to be unchanged except under the mainline metering strategy. In addition, during peak traffic, mainline metering strategy yields the smallest number of lane changes; however, during off-peak traffic, it yields the highest number of lane changes.

It should be noted that excessive lane changing is a key traffic safety hazard. Accordingly, simulation results indicate that under peak traffic conditions, early merge control yields the least hazardous merge control strategy, while late merge control strategy is the most favorable under off-peak traffic conditions. It is also observed that all strategies have consistent impact up-to work zone length of $2000 \mathrm{ft}$ (610 m); however, when work zone length exceeds that limit, significant improvements are observed, indicating that longer work zones yield fewer lane changes.

Speed average (Km/Hour). The link-based Speed Average MOE represents the average speed of vehicles that have completely traversed the link. This MOE was collected for the links occupied by the work zone, in addition to links comprising a stretch of the interstate upstream and downstream of the work zone with the same length. A link speed average index $\left(I_{\mathrm{S}, \text { Link }}\right)$ was calculated to compare the changes in link speed average compared to baseline conditions. Figure 9 illustrates the plot of this $I_{\mathrm{S}, \text { Link }}$ by work zone length for peak and off-peak traffic. A positive value indicates improved speeds, while a negative value indicates slower traffic. 
As showed in Figure 9, all strategies yield better performance under peak traffic compared with off-peak traffic conditions. In addition, temporary ramp metering improved the speed average when implemented, due to its inherent feature of providing priority to mainline vehicles in the expense of those who want to get on the freeway.

Speed average is a key mobility indicator on interstate links, and under such terms temporary ramp metering and mainline metering strategies have the most favorable mobility impact under peak traffic conditions, and early merge control has the most adverse mobility impact under such conditions. However, under off-peak traffic conditions, mainline metering yields the most unfavorable mobility impacts, while all other strategies have minimal impact on mobility. Moreover, it is observed that work zone length has no or minimal impact on speed average under mainline metering strategy. In addition, under temporary ramp metering, longer work zones yield better speed averages. As for late and early merge control, the study results show that longer work zones decreases speed average.

Delay travel per vehicle (Seconds/Vehicle). The link-based MOE delay travel per vehicle represents the average time that vehicles were delayed on the link. A link delay travel per vehicle index $\left(I_{\mathrm{T}, \mathrm{Link}}\right)$ was calculated for the links occupied by the work zone, in addition to links comprising a stretch of the interstate upstream and downstream of the work zone with the same length, to indicate the change in delay travel per vehicle in comparison to baseline conditions. Figure 10 illustrates the plot of $I_{\mathrm{T}, \mathrm{Link}}$ by work zone length for peak and off-peak traffic.

Results aligned with that of link-based speed average reported earlier. More specifically it is observed that all strategies yield better performance under peak traffic compared with off-peak traffic conditions, except for mainline metering where performance worsened under off-peak traffic conditions. In addition, temporary ramp metering minimized delay travel when implemented by providing priority to mainline vehicles in the expense of vehicles merging from the ramp. Delay travel per vehicle complements the speed average MOE as key mobility indicators on interstate links, and under such terms it is observed that temporary ramp metering and late merge control strategies have the least impact on delay travel under peak traffic conditions, and early merge control has the most adverse mobility impact under such conditions. However, under off-peak traffic conditions, mainline metering yields the most unfavorable delays, while all early merge control have the least impact on delay travel. In addition, it is observed that work zone length has no major impact on delay travel.

Emissions total CO $(\mathrm{Grams} / \mathrm{Km})$. The link-based MOE emissions total CO, in grams/Km, represents the total CO emissions per mile produced by all vehicles on the link. This MOE is the key environmental impacts indicator, and was collected for the links occupied by the work zone, in addition to links comprising a stretch of the interstate upstream and downstream of the work zone with the same length. An emissions total CO index $\left(I_{\mathrm{E}, \mathrm{Link}}\right)$ was calculated to indicate the change of emissions in comparison to baseline conditions. Figure 11 illustrates a plot of $I_{\mathrm{E}, \mathrm{Link}}$ by work zone length for peak and off-peak traffic. A positive surge factor indicates worse emissions, and a negative value indicates improvement.

For all strategies except mainline metering, tested merge control strategies resulted in a change of $\pm 10 \%$ of preconstruction values. However, mainline metering resulted in an increase of $70 \%-80 \%$ compared to preconstruction conditions. As expected, all strategies yield better environmental performance under off-peak traffic except for late and early merge controls due to decreased speeds and hence lesser engine loads. In addition, early merge control minimized emissions total $\mathrm{CO}$ when implemented both under peak and off-peak traffic conditions. In addition, it is observed that longer work zones yield better emissions total $\mathrm{CO}$, which indicates that the longer the work zone the more favorable the environmental impacts.

\subsection{Network-Based MOEs}

Network-based MOEs provide a global understanding of the tested merge control strategies at interstate work zones. Two MOEs were selected to describe the mobility impacts of tested merge control strategies, namely speed average and delay travel total. The following sub-sections discuss these two MOEs and relevant observations. Surge Factors were used once again to indicate the change for each network MOE with respect to implemented strategy by work zone length.

Speed Average (Km/Hour). The network-based Speed Average MOE represents the average speed of vehicles traveling in the network. A network speed average index $\left(I_{\mathrm{S}, \mathrm{Net}}\right)$ was calculated to illustrate the percentage change of all vehicles speed average from baseline conditions. Figure 12 illustrates the plot of this $I_{\mathrm{S}, \mathrm{Net}}$ by work 
zone length for peak and off-peak traffic conditions. As mentioned earlier, a positive surge factor indicates lower network speed average and a negative value indicates faster network traffic.

It was observed that all strategies yield better performance under off-peak traffic compared to peak traffic conditions. In addition, under peak traffic conditions, mainline metering had the least impact on the network average speed, while temporary ramp metering had the worst impact. Vehicles speed was reduced by $25 \%-45 \%$ (36 - 27 mph respectively) when merge control strategies were implemented on a work zone. As for off-peak traffic conditions, results indicate that nearly all strategies had no to minimal impact on the network speed average. It is also observed that work zone length had minimal to no impact on the network speed average.

Delay travel total (Hours). The network-based delay travel total MOE represents the total time that all vehicles were delayed in the network. A network delay travel total index $\left(I_{\mathrm{T}, \mathrm{Net}}\right)$ was calculated to illustrate the percentage change of delay travel total from baseline conditions. Figure 13 illustrates a plot of $I_{\mathrm{T}, \mathrm{Net}}$ by work zone length for peak and off-peak traffic.

As expected, all strategies yield better performance under off-peak traffic compared to peak traffic conditions. In addition, under peak traffic conditions, results were consistent with the network speed average as mainline metering had the least impact on the delay travel total, while temporary ramp metering had the worst impact. Delay travel total surged by $100 \%$ - 300\% (equivalent to 28 - 55 seconds/miles traveled, respectively) when merge control strategies were implemented on a work zone during peak periods. Same behavior was observed under off-peak traffic conditions with a surge ranging between $50 \%$ - 225\% (equivalent to 20 - 45 seconds/miles traveled, respectively). It was also observed that work zone length had minimal to no impact on the delay travel total.

\section{Conclusions}

Four merge control strategies for interstate work zones of various lengths were tested in the CORSIM microsimulation platform, namely late merge control, early merge control, mainline merge metering, and temporary ramp metering. The simulation model accounted for the MUTCD requirements as well as driver/vehicle behavior in work zones and considered peak- and off-peak traffic conditions. Five link-based MOEs and two network-based MOEs were analyzed and reported.

Results indicated that under peak traffic conditions the mainline merge metering strategy is expected to yield the most favorable safety impact represented by fewer lane changes, favorable mobility impacts represented by lesser speed reductions, and improved LOS represented by lower densities. However, mainline merge metering would result in undesirable impacts on the environment, represented by higher emissions total CO. Under offpeak traffic conditions, late merge control surpassed all other strategies and yielded the most favorable impacts, except for density, where temporary ramp metering resulted in similar impact on LOS. Moreover, results indicate that under peak traffic conditions several strategies would yield acceptable impacts. Hence, this reveals that nighttime scheduling of interstate maintenance works may not be the most favorable option.

Overall, this study provides practitioners and researchers a comprehensive and comparative evaluation of the four most utilized merge control strategies, under both peak and off-peak traffic conditions within a work zone setting. In addition, the study provides comprehensive information on key parameters to calibrate and setup CORSIM in order to model work zone conditions at freeway facilities. One of the contributions of this work is to explore and test creative ways to model the strategies within the constraints of the CORSIM simulation platform.

Given the findings of this study, it is highly recommended to reexamine scheduling practices of interstate work zones in a future study. Such a study should consider measures for LOS, user satisfaction, and environmental impacts resulting from implemented merge control strategy and choice of scheduling periods. In addition, additional work is recommended to evaluate merge control strategies for work zones present under different configurations such as two-lane freeways and arterial settings.

\section{References}

[1] Wei, H., Pavithran, M., Yi, P., Yang, Q. and Zeng, Q.-A. (2010) Synthesis of Unconventional Dynamic Merge Metering Traffic Control for Work Zones. Open Transportation Journal, 4, 52-60. http://dx.doi.org/10.2174/1874447801004010052

[2] Chitturi, M.V., Benekohal, R.F. and Kaja-Mohideen, A.-Z. (2008) Methodology for Computing Delay and User Costs 
in Work Zones. Transportation Research Record, 2055, 31-38. http://dx.doi.org/10.3141/2055-04

[3] Tang, Y. and Chien, S.I.-J. (2008) Scheduling Work Zones for Highway Maintenance Projects: Considering a Discrete Time-Cost Relation. Transportation Research Record, 2055, 21-30. http://dx.doi.org/10.3141/2055-03

[4] McCoy, P.T. and Pesti, G. (2001) Dynamic Late Merge-Control Concept for Work Zones on Rural Interstate Highways. Transportation Research Record, 1745, 20-26. http://dx.doi.org/10.3141/1745-03

[5] Federal Highway Administration (FHWA) (2012) Manual on Uniform Traffic Control Devices (MUTCD). 2009 Edition, FHWA, Washington DC.

[6] Ramadan, O.E. and Sisiopiku, V.P. (2015) Bottleneck Merge Control Strategies for Work Zones: Available Options and Current Practices. Open Journal of Civil Engineering, 5, 428-436. http://dx.doi.org/10.4236/ojce.2015.54043

[7] Hardy, M. and Wunderlich, K.E. (2009) Traffic Analysis Tools Volume IX: Work Zone Modeling and Simulation—A Guide for Analysts. Report № FHWA-HOP-09-001. Federal Highway Administration, Washington DC.

[8] Alabama Department of Transportation (ALDOT). Alabama Traffic Data, 2014. http://algis.dot.state.al.us/atd/

[9] Dowling, R., Skabardonis, A. and Alexiadis, V. (2004) Traffic Analysis Toolbox Volume III: Guidelines for Applying Traffic Microsimulation Software. Report № FHWA-HRT-04-040. Federal Highway Administration, Washington DC.

[10] Holm, P., Tomich, D., Sloboden, J. and Lowrance, C. (2007) Traffic Analysis Toolbox Volume IV: Guidelines for Applying CORSIM Microsimulation Modeling Software. Report № FHWA-HOP-07-079. Federal Highway Administration, Washington DC.

[11] Schroeder, B.J., Cunningham, C.M., Findley, D.J., Hummer, J.E. and Foyle, R.S. (2010) Manual of Transportation Engineering Studies. 2nd Edition, Institute of Transportation Engineers, Washington DC.

[12] Shoaib, L. (2002) Calibration of FRESIM for Work Zones Using Field Observable Parameters. Master of Science Thesis, University of Cincinnati, Cincinnati.

[13] Vadakpat, G., Stoffels, S. and Dixon, K. (1999) Calibration and Validation of CORSIM for Work Zones. ITE Journal, 69, 12-14.

[14] American Traffic Safety Services Association (ATSSA) (2008) Maintenance Work Zone Safety: Pocket Guide of MUTCD Guidance on Temporary Traffic Control. ATSSA, Fredericksburg.

[15] Zegeer, J.D., Vandehey, M., Blogg, M., Nguyen, K. and Ereti, M. (2008) Default Values for Highway Capacity and Level of Service Analyses. Transportation Research Board, Washington DC. http://worldcat.org/isbn/9780309099318 http://dx.doi.org/10.3141/2071-05

[16] Federal Highway Administration (FHWA) (2015) HPMS Field Manual-Appendix N: Procedures for Estimating Highway Capacity, 2011. http://www.fhwa.dot.gov/ohim/hpmsmanl/appn.cfm

[17] McTrans Center (2011) CORSIM Users Guide. University of Florida, Gainesville.

[18] Transportation Research Board (TRB) (2010) Highway Capacity Manual 2010. TRB, Washington DC. 